\title{
SELBSTORGANISATION VON VORHERSAGEMODELLEN
}

\author{
Johann-Adolf Müller, Hochschule für Technik und wirtschaft Dresden
}

Erfahrungen bei der Analyse und Vorhersage komplizierter Prozesse u.a. in Ökonomie, ökologie und Meteorologie haben gezeigt, daß viele der üblichen Voraussetzungen auf der Grundlage der tatsächlich vorhandenen A-priori-Information kaum oder gar nicht überprüft werden können. Eine praktikab!.e Modellbildung muß deshalb Elemente des interaktiven Modellentwurfs auf der Grundlage der theoretischen und experimentellen Systemanalyse mit Elementen der automatischen Modellerstellung verbinden, wobei die vorhandene Unbestimmtheit bei der Wahl der geeigneten Modelle und ihrer struktur durch parametrische bzw. nichtparametrische Auswahlalgorithmen vermindert werden kann.

Die zur Selbstorganisation mathematischer Modelle entwickelten Algorithmen beruhen auf Prinzipien (Aufteilung der Beobachtungen in Lern- und Prüffolge, Generierung von Hypothesen (Mutationen), Selektion), die auch von Neuronalen Netzen genutzt werden. Im Unterschied zu Neuronalen Netzen, die durch assoziativen Wissenserwerb in der Trainingsphase ein internes Modell aufbauen, ermöglichen die entwickelten Algorithmen zur Selbstorganisation die Einbeziehung vorhandener A-priori-Information in den Auswahlprozeß sowie di‘ explizite Ermittlung von Modellen optimaler Kompliziertheit. Bei geeigneter Wahl der Basisfunktionen können diese Modelle analytisch angegeben werden. Bei der Anwendung der Algorithmen auf unterschiedliche Datensätze in Ökonomie, Ökologie und Meteorologie hat sich ein zweistufiges Herangehen als zweckmäßig erwiesen:

- Nucleus-Bildung mit Hilfe nichtparametrischer Auswahlalgorithmen, d.h. Aufteilung der systemgrößen in disjunkte Teilmengen ähnlicher (bezüglich zeitreihenmuster oder Dynamik) systemgrößen;

- Anwendung parametrischer Auswahlalgorithmen zur Ermittlung dynamischer Modelle (z.B. lineare oder nichtlineare Differenzengleichungssysteme) für die Repräsentanten dieser Teilmengen.

Große Aufmerksamkeit muß der Validierung der erhaltenen Modelle gewidmet werden, d.h. der Überprüfung, ob das ermittelte Modell optimaler Kompliziertheit in der Lage ist, kausale Zusammenhänge zwischen Eingangsgrößen und Ausgangsgrößen zu erfassen. Entsprechend geeignete Testgrößen wurden ausgehend vom Prinzip der Randomisierung begründet und mit Monte-Carlo-simulation ermittelt. Alternativ werden nichtparametrische Auswahlalgorithmen zur Vorhersage angewendet, die auch für kombinatorische Vorhersagen genutzt werden können. 